\title{
On the relation between auditory spatial attention and auditory perceptual asymmetries
}

\author{
T. A. MONDOR and M. P. BRYDEN \\ University of Waterloo, Waterloo, Ontario, Canada
}

\begin{abstract}
Laterality investigators have typically interpreted any perceptual asymmetry as a direct expression of the functional organization of the brain. However, many other confounding factors, including the asymmetric distribution of attention, may also contribute to either the magnitude or the direction of any of these advantages. In two experiments, attention was manipulated in a dichotic listening paradigm by presenting a preexposural tone cue to the ear from which the subject was required to report. The time available to orient attention was manipulated by varying the time period between the onset of the cue and the onset of the trial (stimulus onset asynchrony, or SOA). Results indicated that a right ear advantage for the identification of verbal material obtained at a 150 -msec SOA was almost completely eliminated at an SOA of $450 \mathrm{msec}$. In addition, the direction of the ear advantage for emotion identification was found to depend on task difficulty. A left ear advantage, apparent when task difficulty was minimal, was reversed to a right ear advantage when difficulty was increased. These data are taken as evidence that, when subjects are faced with a difficult dichotic task, there is a general tendency for right-handed subjects to bias their attention toward the right ear. Such a tendency is shown not only to have likely seriously compromised the results of past investigations of functional perceptual asymmetries but also to be inconsistent with previously proposed theories of dichotic listening per. formance.
\end{abstract}

Investigations of the relationship between auditory perceptual asymmetries and hemispheric functional differentiation have typically been performed with the use of a dichotic listening technique wherein two different channels of information are simultaneously presented, one to each ear. The subject is normally required to report, after each of many trials, as much of the presented information as possible. A performance asymmetry favoring the right ear (RE) or left ear (LE) is regularly taken as evidence of a functional superiority of the contralateral hemisphere (see, e.g., Bryden, 1988; Kimura, 1961a). Since information presented to each ear is transmitted to both the ipsilateral and the contralateral hemispheres, such a straightforward interpretation implicitly assumes that the contralateral auditory pathway dominates the ipsilateral pathway. Reassuringly, several pieces of evidence support such an assumption. First, dichotic studies of braindamaged patients with lesions localized to one hemisphere often indicate that there is a deficit in identification performance for the ear contralateral to the lesion site (e.g., Goodglass, 1967; Kimura, 1961b). A second piece of evidence arises from dichotic listening studies of split-brain patients (people in whom the corpus callosum has been severed-usually to stop epileptic seizures). When verbal information is presented dichotically and a verbal re-

This research was supported by a Natural Sciences and Engineering Research Council grant to M. P. Bryden. We would like to thank Albert Bregman, Lester Krueger, Bruno Repp, and Ralph Beitel for their thoughtful review of the manuscript. Address correspondence to T. A. Mondor at the Department of Psychology, McGill University, Montreal, PQ, Canada H3A 1Y1. sponse is required, these patients typically report hearing virtually nothing in the LE while identifying sounds presented to the RE quite well (see Sidtis, 1988, for a review).

Evidence indicating the dominance of the contralateral pathway has been neatly complemented by findings linking ear asymmetries on a dichotic listening test to hemispheric localization of language function. For example, several authors have replicated Kimura's (1961a) observation that people with language localized in the left hemisphere generally identify verbal material better with the RE than with the LE, whereas the reverse is usually true for people with language localized in the right hemisphere (e.g., Zatorre, 1989). Clearly, if one is interested purely in the prediction of hemispheric lateralization of speech processes, then no interpretation of this relation need be offered-it is enough that there is a strong, reliable correlation. No matter how alluring, however, it may be incorrect to interpret this correlational association as indicative of a causal connection between hemispheric functional capability and auditory perceptual asymmetries. It is possible that ear advantages may be the consequence of the interaction of several factors, only one of which is the functional organization of the brain. Thus, if the underlying cause of the correlation between perceptual and brain asymmetries is of primary interest, more critical analyses must be conducted.

While most physiological assessments indicate that language functions are localized in the left hemisphere in about $95 \%$ of right-handed people, dichotic listening studies typically find that only about $80 \%$ of right-handers 
have a right ear advantage (REA) for identifying verbal material. The failure of the dichotic test to categorize all subjects correctly has been interpreted as indicative of the influence of confounding factors. Thus, Sidtis (1982, 1984,1988 ) has argued that part of the inaccuracy of the dichotic listening method is due to individual differences in the strength of the subcortical ipsilateral and contralateral pathways. Sidtis formulated this proposal in order to interpret the observation that, in his original study (Sidtis, 1982), only about half of his right-handed subjects showed the expected REA for speech identification and left ear advantage (LEA) for pitch perception. The other half of his subjects performed better on both tasks with the same ear. Moreover, for subjects with a consistent ear advantage, the size of the perceptual advantage in the expected direction was significantly larger than that in the unexpected direction. Sidtis (1988) interpreted these data as an indication that

there was a directional bias influencing perceptual asymmetries due to specialized left and right hemisphere processing that was greater in the subjects who showed a single reversal of asymmetry than in subjects who showed both of the "expected" asymmetries. When this bias produced a reversal on one of the tests, it also produced an exaggeration of the expected result on the other test. (p. 169)

While these data are consistent with Sidtis's (1982) notion of subcortical pathway asymmetries, they are also clearly compatible with a consistent attentional bias to one side of space. ${ }^{1}$ According to this view, the attentional bias may act either to magnify or to attenuate a natural ear advantage.

In 1978, Bryden argued that uncontrolled subject strategies such as the deployment of attention may contribute to the perceptual asymmetries observed in laterality studies. To address this problem, he recommended that experimenters use procedures that would restrict the influence of these unwanted variables. In particular, Bryden advocated the use of a dichotic listening procedure wherein subjects are directed to pay attention to, and report from, only one ear on each trial. The logic of such a forced-attention procedure is that if subjects are forced to attend to a specified ear for a block of trials, any natural attentional bias should be eliminated, or at least reduced. A comparison of RE performance when the RE is attended and LE performance when the LE is attended should, therefore, provide a measure of perceptual asymmetries relatively uninfluenced by attentional biases.

The results of studies done with the forced-attention procedure have demonstrated that attention can alter performance in a dichotic task, since people typically perform better on the attended than on the unattended ear (e.g., Bryden, Munhall, \& Allard, 1983). Reports of an influence of attention on the magnitude of perceptual asymmetries are, however, quite rare (e.g., Hiscock \& Stewart, 1984; Hugdahl \& Andersson, 1986). Rather more typical are reports that ear advantages of comparable magnitude are obtained whether these advantages are assessed with the standard divided-attention or the forced-attention technique (e.g., Bloch \& Hellige, 1989; Bryden et al., 1983; Bryden \& Murray, 1985; Dean \& Hua, 1982; Obrzut, Conrad, Bryden, \& Boliek, 1988). The greater relative frequency with which null findings have been reported has led many experimenters to reject attentional factors as a major contributor to ear asymmetries. Clearly this may be an inappropriate interpretation, since the evidence allows only that, for the particular manipulation undertaken, no significant effect of attention was found. This, in turn, may indicate either that attentional factors are truly irrelevant or that the forced-attention technique employed to control auditory attention is inadequate.

Recently, Mondor and Bryden (1991) used a precuing paradigm popular in studies of visual attention to explore the prospect that attentional factors influence the magnitude or direction of the typical dichotic REA for the identification of consonant-vowel (CV) syllables. Attention was manipulated by sounding a tone, prior to the beginning of each trial, in the ear from which the subject was to report. The subject's task was to report only the syllable that had been presented to the cued ear. The time between the onset of the tone and the onset of the syllables (stimulus onset asynchrony, or SOA) was varied (150, 450 , or $750 \mathrm{msec}$ ) to control the amount of time available for the subject to orient attention to the cued ear. The outcome of the study revealed that attentional factors are, indeed, important, since the magnitude of the REA declined substantially as SOA increased (the initial REA of $11 \%$ at the 150 -msec SOA was reduced to $3 \%$ at the $450-$ msec SOA). Interestingly, this reduction was primarily attributable to an improvement in LE but not RE performance. Thus, it was tentatively concluded that such a pattern of results was consistent with the notion that attention is initially biased to the RE and that the lateralized tone cue goes some way toward overcoming this bias. In a subsequent series of experiments, Mondor and Bryden (1992b) showed, by comparing facilitation and inhibition for valid and invalid trials, that the lateralized cue exerts its effect by drawing attention to the ear in which it sounds, not by alerting the subject about the forthcoming trial.

One reason that the precuing technique has been more successful than the forced-attention method in identifying a significant contribution of attention to the magnitude of perceptual asymmetries may well lie in the different types of cues used to orient attention. Two different types of cues are commonly used to orient visual attention. Exogenous or pull cues are presented in a location spatially proximate to the location of the forthcoming stimulus. Alternatively, endogenous or push cues (often an arrow) indicate the location where the stimulus will be presented, but do so from a distinctly separate spatial location. Recently, Yantis and Jonides (1990) have shown that pull cues usually automatically capture attention (as long as subjects consciously have no conflicting strategy), whereas push cues must be consciously processed before attention is oriented. Indeed, Müller and Rabbitt's (1989) conclusion that there are "distinct but interacting reflexive and voluntary orienting mechanisms"' (p. 315) lends support to this notion. To extend the findings of these 
visual studies, the verbal cues typically used in forcedattention dichotic experiments may be, in essence, push cues that can act to orient attention only with the subject's cooperation. The dependence of this type of cue on cooperation might allow interference by idiosyncratic subject strategies. ${ }^{2}$ The orienting potential of the lateralized tone cue, on the other hand, may be less influenced by subject strategies in that it may act as a pull cue, which usually automatically draws the subject's attention to the appropriate ear on every trial.

It was not apparent from Mondor and Bryden's (1991; 1992b) studies whether or not attention is consistently biased toward the RE for all types of auditory processing. Clearly, demonstration of such a consistent bias would go some way toward explaining the relatively less frequent and more labile LEAs reported in the literature (Bryden, 1982). Alternatively, the direction of an attentional advantage may depend on the type of processing required of the subject. Such a notion is consistent with Kinsbourne's (1975) attentional theory of perceptual asymmetries. According to this model, ear advantages arise because of an activational advantage in favor of the hemisphere specialized to perform the task in which the subject is engaged. In Kinsbourne's view, one by-product of such an activational asymmetry would be an attentional bias to the contralateral region of auditory space.

The following experiments were designed to determine whether auditory attention is biased toward the RE for tasks better performed by the left hemisphere and to the LE for tasks better performed by the right hemisphere. To this end, the precuing technique employed by Mondor and Bryden (1991) was used to orient attention for both an emotion identification task and a word identification task. Bryden and MacRae (1988) and Bryden, Free, Gagne, and Groff (1991) have shown that these tasks produce an LEA and an REA, respectively. In addition, both of these ear advantages complement neuropsychological findings indicating that the right hemisphere is specialized for the perception and identification of emotion, whereas the left hemisphere is specialized for the perception and identification of verbal material (Bryden, 1982).

\section{EXPERIMENT 1}

The first experiment was performed to determine whether the direction of an attentional bias is dependent on the type of processing required. The words bower, dower, power, and tower, each pronounced with an angry, happy, neutral, or sad tone of voice were used as stimuli (cf. Bryden et al., 1991; Bryden \& MacRae, 1988). A tone cue was presented to one ear immediately prior to each dichotic trial. Subjects were asked to report either the emotional expression of the word or the word itself pronounced in the ear in which the cue sounded. The time between the onset of the cue and the onset of the stimuli was varied to manipulate the amount of time available for the subject to orient attention. It may be that the direction of an attentional bias is dependent on the type of processing required, in such a way that subjects pay relatively more attention to the $\mathrm{RE}$ when asked to identify speech sounds but pay more attention to the LE when asked to identify emotions. If such a dependency exists, an initially large LEA for emotion identification and REA for word identification should be reduced as SOA and the opportunity to orient attention increase, since the attentional bias will be attenuated. Alternatively, attention may be consistently biased to the RE. If such a consistent RE bias exists, then, as in the first model, an initially large REA for word identification should be reduced as SOA increases. The pattern of performance for the emotion identification task should, however, be quite different. An initially small LEA for the identification of emotion should be enlarged as SOA increases, since, in response to the cue, attention will be drawn away from the RE and redistributed to the LE.

\section{Method}

\section{Subjects}

Twenty-four undergraduate students, 12 male and 12 female, were paid for their participation. All subjects were right-handed according to self-report. None of the subjects reported any hearing impairment and all spoke English as their first language. Half of the males and half of the females completed the emotion task, while the other half of the subjects completed the word task.

\section{Materials}

The stimuli were the words bower, dower, power, and tower spoken in a male voice, each of which was pronounced with angry, happy, neutral, and sad expressions of emotion. ${ }^{3}$ The 16 sounds were digitized, and their volumes equalized, on a modified PDP11/40 computer and edited to a duration of $500 \mathrm{msec}$. Each trial was preceded by a computer-generated $100-\mathrm{msec}, 600-\mathrm{Hz}$ sine-wave tone. The stimuli were recorded onto an audio tape and played back to the subjects on a Sony TC - R303 tape recorder with Dolby noise reduction. The subjects listened to the tape through Koss Pro/4x Plus headphones at $70 \mathrm{~dB}$ SPL. The peak intensities of sounds recorded on the right and left channels were equalized by using a Bruel \& Kjaer Impulse Precision sound level meter. The headphones were reversed for half of the subjects.

\section{Design and Procedure}

On each trial, two different words were presented simultaneously -one to each ear. A tone was presented to either one ear or the other prior to every trial. This tone was designed to cue subjects to focus attention on the ear in which it sounded. The opportunity to orient attention was manipulated by controlling cue-trial SOA. The subjects were allowed $3.5 \mathrm{sec}$ to respond to each trial and to ready themselves for the beginning of the next trial.

The subjects were given 12 sample trials identical to the ones they would be faced with in the experiment, except that the stimuli were CV syllables instead of words spoken with different emotional expressions. In addition, each of the 16 sounds ( 4 words $\times 4$ emotions) was played twice for the subjects so that they could become acquainted with the experimental stimuli prior to the experiment.

Each of the 16 stimuli could be paired with 9 others (given that neither the same affect nor the same word was presented to both ears on the same trial). Thus, there were 144 unique stimulus combinations. For each of these 144 stimulus combinations, either the right or the left ear could be cued at one of three different SOAs. Thus, since this design resulted in 864 unique trial combinations, a random selection of trials was used. Stringent controls, however, ensured that each emotion and each word was presented an equal number of times in each ear at each SOA. Each of the 16 stimuli 
(word $\times$ emotion) was presented nine times when the LE was cued-three times at each of three SOAs $(150,450$, and $750 \mathrm{msec})-$ and nine times when the RE was cued-three times at each of the three SOAs. Within each of the six 48-trial blocks, the subjects were cued to attend to each ear eight times at each SOA. Presentation order was essentially random, with the constraint that one ear was cued no more than four times in a row. The subjects completed a total of 288 trials. A short respite was provided on completion of each block of 48 experimental trials. The subjects were asked to indicate the correct response by circling, on a response sheet, the word/emotion presented to the ear in which the cue had sounded.

\section{Results \\ Emotion Identification}

Percent errors. A two-way (ear $\times$ SOA) analysis of variance (ANOVA), in which percent errors were the dependent variable, revealed significant main effects of ear $[F(1,11)=5.13, p<.05]$ and of SOA $[F(2,22)=16.54$, $p<.01$ ]. Thus, as is shown in Figure 1 (top panel), subjects performed better with the LE than with the RE, while performance generally improved with increases in SOA.

The ear $\times$ SOA interaction, however, failed to reach significance $(F<1)$. No evidence was obtained, therefore, to support the notion that when subjects expect to identify emotions there is an attentional bias toward the LE. Neither was any evidence found to indicate that there was an attentional bias toward the RE. Rather, the precuing technique appears to have effectively controlled subjects' strategies in such a way that attention was not preferentially directed toward either ear.
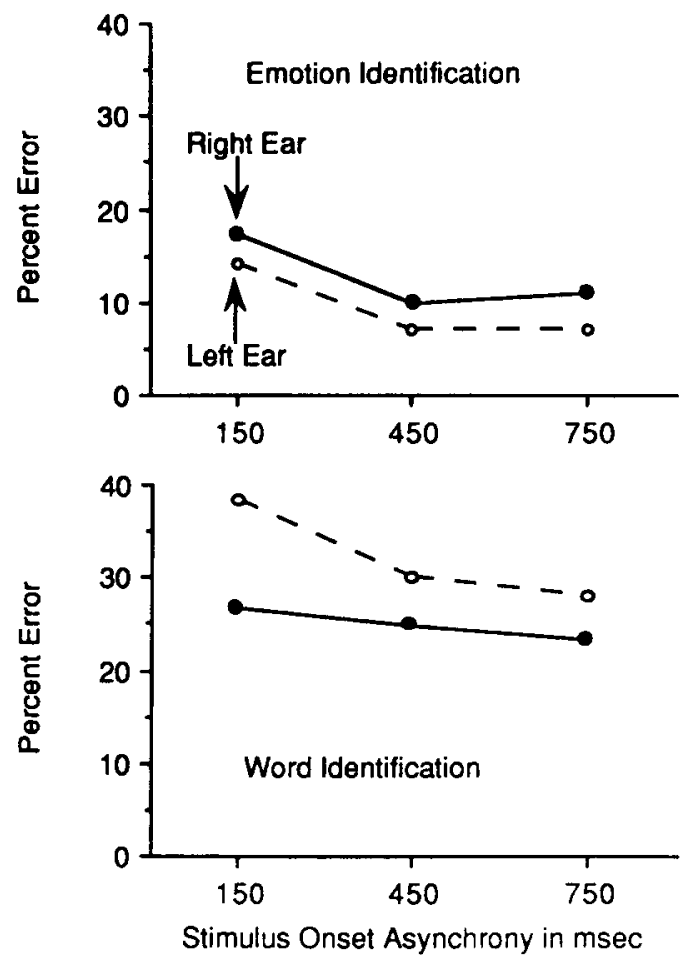

Figure 1. Percent error for emotion (top panel) and word (bottom panel) identification as a function of stimulus onset asynchrony for the right and left ears (Experiment 1).
It is important to note that the magnitude of the LEA is, at $3 \%$, extremely small and, in fact, is much smaller than the $12 \%$ and $11 \%$ advantages reported by Bryden and MacRae (1988) and by Bryden et al. (1991). This difference in the magnitude of the perceptual asymmetry is not simply a statistical artifact due to the difference in error rates, since Bryden and Sprott's (1981) lambda (which corrects for overall error rate) also reveals a much smaller laterality effect in the present experiment $(0.31)$ than in either of the two previous studies (0.70 and 0.87 in Bryden \& MacRae, 1988, and in Bryden et al., 1991, respectively). However, the much better average performance of the subjects in the present experiment (11\% errors) than of those who participated in either of the earlier investigations (28\% errors in both studies) may have created a ceiling effect that acted not only to reduce the ear advantage but also to mask any effect of attention that might normally be present. This difference in accuracy may be a reflection of the different techniques used in the studies. Whereas Bryden and MacRae (1988) required subjects to monitor both ears and determine whether a prespecified target had been presented in either ear on each trial, in the present experiment subjects were required to pay attention to, and report from, only one ear on each trial. Thus, rather unsurprisingly, it appears that having to monitor both ears simultaneously makes the task more difficult than does monitoring only one ear at a time.

Subjects can make two different types of errors. They may correctly report the stimulus presented to the uncued ear (an intrusion error) or they may report a stimulus that was not presented to either ear (a miss error). Analysis of the relative proportion of these two different types of errors at each of the three SOAs allows a further assessment of the effect of the cue. Assume that subjects tend to report the one of the two presented stimuli that seems most salient to them. Assume further that the most salient stimulus tends to be that which is attended. If the cue is "completely" effective in drawing attention to the cued ear in such a way that no information from the uncued ear is available, then; on those trials on which an error is made, the probability of reporting any one of the stimuli not presented to the cued ear is $1 / 3$ (recall that there are only 4 stimuli in total). One of these responses will be classified as an intrusion error, while the other two will be classified as miss errors. Thus, given that an error is made, the probability of making an intrusion error will be $1 / 3$, and the probability of making a miss error will be $2 / 3$. If the cue is less than "completely effective," attention will be occasionally focused on the uncued ear and information from that ear will become available to the subject. Given these circumstances, the likelihood of making an intrusion error will increase (the likelihood of making a miss error will decrease) relative to that apparent when attention is confined to the cued ear. This rationale, therefore, predicts that as the cue's effectiveness increases, the proportion of intrusion errors should decrease while that for misses should increase. If these predictions are supported, then some converging evidence will be obtained in support of Mondor and Bryden's 
Table 1

Percent Intrusions and Percent Misses for Emotion and Word Identification as a Function of Stimulus Onset Asynchrony and Ear for Experiments 1 and 2

\begin{tabular}{|c|c|c|c|c|c|c|}
\hline & \multicolumn{6}{|c|}{ Stimulus Onset Asynchronies (in Milliseconds) } \\
\hline & \multicolumn{3}{|c|}{ Left Ear } & \multicolumn{3}{|c|}{ Right Ear } \\
\hline & 150 & 450 & 750 & 150 & 450 & 750 \\
\hline \multicolumn{7}{|c|}{ Experiment 1: Emotion } \\
\hline Percent intrusions & .78 & .39 & .48 & .77 & .20 & .61 \\
\hline Percent misses & .22 & .61 & .52 & .23 & .80 & .39 \\
\hline \multicolumn{7}{|c|}{ Experiment 1: Word } \\
\hline Percent intrusions & .70 & .61 & .63 & .73 & .50 & .57 \\
\hline Percent misses & .30 & .39 & .37 & .27 & .50 & .43 \\
\hline \multicolumn{7}{|c|}{ Experiment 2: Emotion } \\
\hline Percent intrusions & .63 & .23 & .57 & .62 & .30 & .67 \\
\hline Percent misses & .37 & .77 & .43 & .38 & .70 & .33 \\
\hline \multicolumn{7}{|c|}{ Experiment 2: Word } \\
\hline Percent intrusions & .65 & .43 & .65 & .72 & .57 & .63 \\
\hline Percent misses & .35 & .57 & .35 & .28 & .43 & .37 \\
\hline
\end{tabular}

(1992b) conclusion that the lateralized cue acts to orient attention to the ear in which it sounds. Since percent misses are the complement of percent intrusions (percent errors $=1$ - percent intrusions), analyses for the two measures are identical. Therefore, only percent intrusions were analyzed.

Percent intrusions. A two-way ANOVA (ear $\times$ SOA), with percent intrusions as the dependent variable, revealed only a significant main effect of SOA $[F(2,22)=14.49$, $p<.01]$. Percent intrusion errors decreased significantly from the $150-\mathrm{msec}$ to the $450-\mathrm{msec}$ SOA (p. $<.05$ by Tukey $H S D$ test). Thus, the data conformed to the prediction that percent intrusions should decline as the effectiveness of the cue increased. In accordance with data reported by Mondor and Bryden (1992b), the cue appears to exert its effect by orienting attention to the ear in which it sounds. Apparently, however, the low error rate may have obscured an effect of the cue on overall performance. Neither the main effect of ear $(F<1)$ nor the ear $\times$ SOA interaction $[F(2,22)=1.52, p=.24]$ reached significance. Percent intrusions and percent misses are reported in Table 1.

\section{Word Identification}

Percent errors. The main effects of ear $[F(1,11)=$ $4.77, p=.05]$ and of $\operatorname{SOA}[F(2,22)=13.96, p<.01]$ reached statistical significance. More importantly, however, the ear $\times$ SOA interaction was also significant $[F(2,22)=4.96, p<.01]$. Figure 1 (bottom panel) shows that a strong REA of $12 \%$ at the $150-\mathrm{msec}$ SOA $[F(1,11)=7.96, p<.01]$ was reduced to $5 \%$ at both the 450 -msec $[F(1,11)=1.76, p=.21]$ and the 750msec SOA $[F(1,11)=2.90, p=.12]$. In addition, performance improved for the LE but not for the RE. The form of this interaction is almost identical to the $11 \%$ REA at $150 \mathrm{msec}$ and the $3 \%$ REA at $\mathbf{4 5 0} \mathrm{msec}$ for identifying CV syllables reported by Mondor and Bryden (1991). That the present interaction was obtained with a different task and with a different group of subjects provides con- firmatory evidence for the interpretation that REAs for the identification of verbal material are indeed produced by an interaction between the spatial distribution of attention and hemispheric functional capabilities. Across studies, the average $70 \%$ reduction in the magnitude of the REA demonstrates the enormous inflationary effect that the attentional bias has in creating the REA for the perception and identification of verbal stimuli.

Percent intrusions. A two-way ANOVA (ear $\times$ SOA) was performed, with percent intrusions as the dependent variable. Only the main effect of SOA reached significance $[F(2,22)=9.57, p<.01]$. Percent intrusions, reported in Table 1, declined from the 150 -msec to the 450 msec SOA ( $p<.05$ by Tukey $H S D$ test), indicating that, for word identification as well as for emotion identification, attention is deployed to the cued ear. Neither the main effect of ear $[F(1,11)=1.21, p=.30]$ nor the ear $\times$ SOA interaction $(F<1)$ reached significance.

\section{Discussion}

Mondor and Bryden (1991) proposed that the REAs typically obtained for the identification of verbal material are the product of an interaction between attentional and structural factors. The present investigation provided strong support for this interpretation, since an REA for the discrimination of words at the 150-msec SOA was substantially reduced at the $450-\mathrm{msec}$ SOA. Most important for our present purposes, however, was that no evidence of such a relation was found for the LEA for the perception of emotional tone. This null finding may, of course, have been found for several different reasons. It is possible that the low average error rate on the emotion task (11\%) may have attenuated the LEA and obscured an effect of attention. The validity of this interpretation can be ascertained by determining the effect of task difficulty on the pattern of performance. If the high level of accuracy in Experiment 1 acted to mask an effect of attention, then an increment in task difficulty in Experiment 2 should not only increase the magnitude of the LEA for emotion iden- 
tification but also increase the probability of observing an effect of attention.

It is also possible that the instructions used in the present study, which forced subjects to attend to only one ear on each trial, acted to eliminate subject strategy factors that accounted for most of the LEA obtained by Bryden and his colleagues (Bryden et al., 1991; Bryden \& MacRae, 1988). Neither Bryden and MacRae (1988) nor Bryden et al. (1991) attempted to control the strategy effects that may have contributed to the LEA for the identification of emotional tone that they reported. For example, subjects may have biased their attention to the LE and/or analyzed first the left and then the right channels before responding. Clearly, adoption of either, or both, of these strategies could have contributed to their results to an unknown extent. Whatever the specific mechanism, if the LEA is predominantly a result of subject strategies, an increase in task difficulty should have relatively little influence on the magnitude of the LEA since the precuing technique will again restrict the opportunity for subjects to employ such strategies.

Finally, it may be that an attentional bias toward the RE occurs only when the subject perceives the task to be difficult. This possibility stems from the observation that effects of attention on the REA for CV (Mondor \& Bryden, 1991) and word (Experiment 1 of the present study) identification have been observed only when errors have averaged about $25 \%$. Some of the data reported by Kimura (1961a) provide supporting evidence for the possibility of an attentional bias. In Kimura's study, people in whom language was localized to the left hemisphere (as shown by sodium amytal testing) identified spoken digits better with the RE than with the LE. The reverse effect was observed for subjects with right hemisphere language. Intriguingly, however, right-handed subjects with left hemisphere language had larger REAs than did left-handed subjects with left hemisphere language. A similar effect of handedness-lateralization consistency was observed for subjects with right hemisphere language (see also Zatorre, 1989, for a similar effect). As Bryden (1978) has noted, this effect demonstrates that "handedness has an effect on dichotic listening performance over and above that of speech lateralization"' (p. 125). Clearly, it is possible that this is an attentional effect. That is, the very fact that someone is right-handed may be associated with a tendency to attend to the right side of space during a dichotic listening task. Given this apparent tendency, it is possible that an increase in task difficulty in the emotion task would produce an attentional bias toward the RE. If it is true that, once subject strategy effects are controlled, there is only a very small LEA for the perception of emotional tone, we might expect that such an attentional bias will produce an REA for the task. Such a finding not only would confirm that the ear asymmetry for emotional tone is extremely small but also would establish the existence of a general tendency for right-handed subjects to bias attention toward the RE when faced with a difficult dichotic task.

\section{EXPERIMENT 2}

Experiment 2 was conducted to more accurately delineate the mechanism underlying LEAs. To this end, the tape used in the preceding study was used once again but was played at a lower intensity. This manipulation was undertaken on the grounds that a decrease in intensity would cause an increase in the difficulty of the emotion task. As outlined above, such an increase in difficulty should provide data enabling a more refined delineation of the influence of attention on the LEA for emotional tone identification.

\section{Method}

Subjects

Twenty right-handed undergraduate students, 10 of them male and 10 female, were paid for their participation. None of the subjects reported any hearing impairment, and all spoke English as their first language.

\section{Materials}

The materials were identical to those in Experiment 1, except that the audio tape was played to subjects on a Sanyo M-V45 tape deck at $48 \mathrm{~dB}$ SPL.

\section{Design and Procedure \\ The design and the procedure of Experiment 2 were identical to those of Experiment 1.}

\section{Results \\ Emotion Identification}

Percent errors. The reduction in intensity successfully increased the difficulty of the emotion identification task, since the average error rate rose from $11 \%$ in Experiment 1 to $23 \%$ in Experiment $2[t(20)=2.39, p<.05]$. A two-way ANOVA (ear $\times$ SOA) showed that the main effect of SOA reached significance $[F(2,18)=4.47, p<$ $.05]$, and that of ear approached significance $[F(1,9)=$ $4.81, p=.06]$. These effects were compromised, however, by the ear $\times$ SOA interaction, which also attained statistical significance $[F(2,18)=5.07, p<.05]$. As is shown in Figure 2 (top panel), a significant REA of $8 \%$ at the 150 -msec SOA $[F(1,9)=13.88, p<.01]$ was markedly attenuated to $2 \%$ at both the $450-\mathrm{msec}(F<1)$ and the 750 -msec $(F<1)$ SOAs.

This result is theoretically significant for several reasons. First, and most importantly, the reversal in ear advantage from Experiment 1 to Experiment 2 supports the notion that there is a tendency for right-handers to bias their attention to the RE in a difficult dichotic listening situation. The modulation of the REA by the opportunity to orient attention is clearly consistent with the existence of this bias. Second, the absence of any LEA even in a 

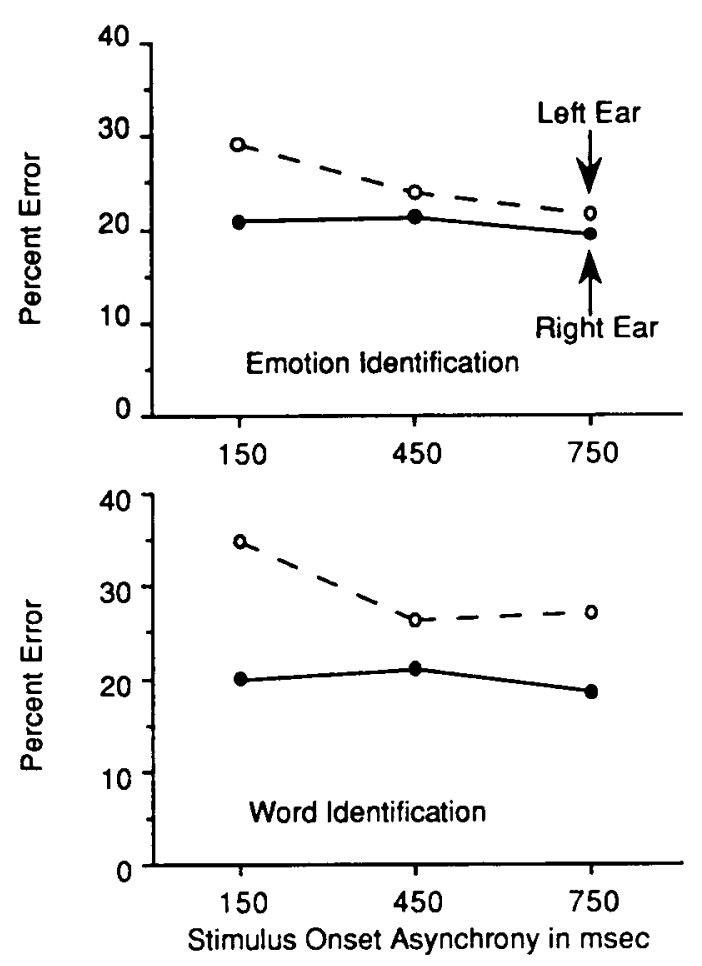

Figure 2. Percent error for emotion (top panel) and word (bottom panel) identification as a function of stimulus onset asynchrony for the right and left ears (Experiment 2).

task more difficult than that in Experiment 1, as well as the nonsignificant ear advantages obtained at SOAs of 450 and $750 \mathrm{msec}$ in both experiments, lends support to the notion that when subjects' strategies are restricted, there is no meaningful LEA for the perception and identification of emotional tone.

Percent intrusions. Analysis of the percent intrusions for the emotion identification task revealed only a significant main effect of SOA $[F(2,18)=42.21, p<.01]$. As in Experiment 1, Table 1 indicates that percent intrusions declined from the 150 -msec to the $450-\mathrm{msec}$ SOA ( $p<.05$ by Tukey $H S D$ test). The main effect of ear $[F(1,9)=1.37, p=.27]$, as well as the ear $\times$ SOA interaction $(F<1)$ both failed to reach statistical significance. This finding replicates a similar effect obtained in Experiment 1 and provides further support for the notion that auditory attention is oriented in response to the cue.

\section{Word Identification}

Percent errors. Rather surprisingly, the mean error rate for word identification in this study was, at $24 \%$, identical to that in Experiment 1. Thus, it appears that verbal information is less degraded by a decrease in intensity than is emotion information. In any event, statistical analysis yielded significant main effects of ear $[F(1,9)=15.27$, $p<.01]$ and of SOA $[F(2,18)=4.79, p<.05]$. These effects were compromised, however, by a significant ear $\times$ SOA interaction $[F(2,18)=7.57, p<.01]$, shown in Figure 2 (bottom panel), which indicated that a large REA of $15 \%$ of the 150 -msec SOA $[F(1,9)=20.73, p<$ $.01]$ was reduced to $5 \%$ at $450 \mathrm{msec}[F(1,9)=2.95, p=$ $.12]$ and $9 \%$ at $750 \mathrm{msec}[F(1,9)=17.02, p<.01]$. Thus, the interaction obtained in Experiment 1 and by Mondor and Bryden (1991) was replicated. The slight increase in the size of the REA at $750 \mathrm{msec}$ has previously been reported by Mondor and Bryden (1991), who demonstrated that it indicated that the $450-\mathrm{msec}$ SOA is the optimal interval for orienting attention in this type of study. Once again, the overwhelming influence of attention on the REA long thought to represent only functional hemispheric differences was demonstrated.

Percent intrusions. The main effect of SOA $[F(2,18)=7.44, p<.01]$, but not that of ear $[F(1,9)=$ $1.98, p=.19$ ], attained statistical significance. In addition, the ear $\times$ SOA interaction was significant $[F(2,18)$ $=4.16, p<.05]$. The effect of SOA was significant for both the $\operatorname{LE}[F(2,18)=3.66, p<.05]$ and the RE $[F(2,18)=16.80, p<.01]$. For both ears, percent intrusions declined from the 150 -msec SOA to the 450 -msec SOA ( $p<.05$ by Tukey HSD test). As is shown in Table 1 , the interaction was due principally to the larger decline in percent intrusions for the LE than for the RE. Thus, once again, evidence was obtained in support of the interpretation that the lateralized tone acts to draw attention to the cued ear.

\section{Discussion}

The attempt to increase difficulty of the emotion task by reducing the intensity in which stimuli were presented was successful. However, no attentional bias toward the LE was apparent for the emotion identification task. Rather, the increase in difficulty engendered an initial REA for the identification of emotional tone. This finding, therefore, constitutes the first evidence that the asymmetric distribution of auditory attention may be completely responsible for the very direction of a perceptual advantage.

In addition, since only a 3\% LEA could be detected in Experiment 1 and since this advantage was not increased but rather reversed when the task was made more difficult in Experiment 2, it would seem most appropriate to conclude that there is, if anything, only a very small LEA for the identification of emotional tone. Thus, the inflated LEAs reported by Bryden and MacRae (1988) and by Bryden et al. (1991) for this task appear to have been due to subjects' strategies, for which there was no control. This failure to replicate a strong LEA for emotion identification neatly complements Bryden's (1978) contention that

if one is concerned primarily with the investigation of cerebral asymmetries in the normal subject, then we must take every precaution to minimize the subject's control over the situation. In particular, the subject cannot be left free to deploy attention as he chooses, but some formal control must be employed. Otherwise, we run the danger of measuring not cerebral lateralization, but how the subject responds to the situation. (p. 143) 


\section{GENERAL DISCUSSION}

Taken together, the results of these two experiments have several important methodological and theoretical implications. Most significantly, the reversal of the LEA for the identification of emotion found in Experiment 1 to an REA in Experiment 2 does not conform with the predictions of either of the previously proposed models of perceptual asymmetries. The more popular structural model (Kimura, 1961a) proposes that any ear asymmetry may be directly related to the lateralization of function within the brain. Such a model obviously cannot account for a reversal in ear advantage simply because of an increase in task difficulty, since, in both cases, the subject must identify emotional tone. Similarly inadequate is Kinsbourne's (1975) attentional model, which predicts that the direction of an attentional bias should depend on the type of processing in which the subject is engaged. Clearly, were this view correct, an attentional bias favoring the LE for emotion identification should have been apparent in both experiments. Rather, the results are consistent with a model that recognizes the existence of a tendency for right-handed subjects to bias attention to the RE under difficult discrimination conditions.

Several authors have argued that attentional factors do not necessarily influence ear asymmetries since LE and RE advantages can be obtained "simultaneously." For example, Ley and Bryden (1982) presented subjects with two different sentences on each dichotic trial. Immediately following presentation of the trial, subjects were asked to make judgments of both the verbal content of the sentence and the emotional tone of the sentence. These authors obtained an LEA for the identification of the emotional tone and an REA for the identification of the verbal content of the sentence. Clearly, this effect cannot be explained by a simple attentional bias toward one ear. The effect is cast in doubt, however, since there have been no published replications of it or, indeed, of other similar effects (see, e.g., Kallman, 1978). In any event, even if accurate, these findings indicate only that attentional factors may be of relatively little importance when subjects are unable to develop a single consistent strategy for performance on any given trial. This is clearly not typically the case in most dichotic listening experiments, since the subjects expect and are presented with only one type of discrimination on each of several consecutive trials.

It has been suggested that the fused rhymed words test (Wexler \& Halwes, 1981, 1983) eliminates any effect of attention on perceptual advantages. Stimuli for this dichotic test are created by pairing the beginning of each word with a composite of the ends of all words. Wexler and Halwes $(1981,1983)$ have reported that, when these stimuli are presented dichotically, they "fuse into a single auditory image" (1983, p. 60). Apparently, because of this phenomenological impression, it is assumed that an attentional bias to one ear is impossible. The only support that Wexler and Halwes give for this assumption is that "subjects expected, experienced and reported only one response on each trial"' (1983, p. 60). To conclude on the basis of this statement alone that attentional factors are unimportant seems unwarranted. Repp's (1976) suggestion that forced-attention instructions have little effect on performance on the fused words test does not necessarily mean that dichotic performance on the test is uninfluenced by the spatial distribution of auditory attention. Rather, as discussed above, the forced-attention technique appears to be an inadequate method of orienting attention. Thus, until proper control experiments (using the precuing technique) are conducted, we must reserve judgment as to the effect of attention on the fused rhymed words test. However, given that we have shown that there is a consistent attentional bias toward the RE, it would be rather surprising if this asymmetric distribution of attention did not influence performance on any dichotic listening test.

In most laterality studies, accuracy is the dependent measure, so subjects make between $20 \%$ and $30 \%$ errors. Evidence obtained from the present study indicates that only when accuracy declines to this level does an attentional bias toward the RE become apparent. However, it seems likely that a similar bias would be produced if subjects were pressured to respond quickly. Thus, because previous studies have failed to control adequately for attentional biases, the obtained ear asymmetries have very likely been governed by the interacting effects of hemispheric specialization and attentional biases.

This interaction can produce ear advantages in either of two ways. Clearly, if perceptual advantages are completely determined by hemispheric asymmetries, any ear advantage should be directly dependent on hemispheric specialization. If, however, attention is also an important determinant, an attentional bias toward the RE may either magnify or reduce any perceptual advantage. Consider, first, tasks for which the left hemisphere is specialized. An attentional bias toward the RE would complement this functional component and, in so doing, increase the magnitude of the REA. Evidence from the present experiments and from Mondor and Bryden (1991) is certainly consistent with such an effect, since REAs for the identification of verbal material were observed to be substantially attenuated when subjects were given sufficient time to orient their attention to the LE.

The interaction between the spatial distribution of auditory attention and functional hemispheric differences can influence auditory asymmetries in a second manner. If the right hemisphere is specialized for a particular task, an attentional bias toward the RE would work in opposition to this functional asymmetry and attenuate, mask, or even reverse the apparent ear advantage. The extent to which the LEA is reduced or reversed would depend on the relative strengths of the functional advantage and the attentional bias. If the functional component is more robust than the attentional bias, an LEA would likely be observed. Alternatively, if the functional component is equal to or weaker than the attentional bias, either no ear advantage at all or an REA will be observed. The data obtained from this study 
indicate that this effect may occur, since a small LEA for the identification of emotional tone apparent in Experiment 1 was reversed to an REA in Experiment 2 because of an attentional bias toward the RE. Thus, ear advantages are not simply a reflection of hemispheric functioning. Rather, it appears that the extent to which hemispheric and attentional components work cooperatively or in opposition dictates the apparent ear advantage.

Finally, the very small ear advantages observed for both emotion identification and word identification (and CV identification in Mondor \& Bryden, 1991) once the contribution of the RE attentional bias is controlled beg the question of whether such differences are theoretically useful. What exactly does it mean that the RE is $5 \%$ better at identifying words and that the LE is $3 \%$ better at identifying emotional tones? This question becomes even more important if the precuing technique employed does not completely eliminate the contribution of attentional biases or subjects' strategies to obtained ear advantages. Thus, the "true" ear advantage may well be even smaller than that reported here and by Mondor and Bryden (1991). At the very least, these small advantages demonstrate that the right and left hemispheres may identify speech sounds, words, and emotional tones almost equally well. A similar equivalence of the left and right hemispheres for processing visually presented verbal material was reported by Mondor and Bryden (1992a), who employed a visual precuing technique to orient attention. In a series of experiments, the frequently reported right visual field advantage for identifying single letters and for making lexical decisions was completely eliminated when attentional resources had been sufficiently and equally allocated to the right and left visual fields.

It is important to note that these perceptual equivalences occur even though extensive neurological data indicate that, in right-handers, the left hemisphere is almost always dominant for language functions, whereas the right hemisphere is typically dominant for spatial functions (see, e.g., Bryden, Hécaen, \& DeAgostini, 1983; Springer \& Deutsch, 1989). If there is any legitimate connection between visual field and ear performance and the capabilities of the contralateral hemisphere, it seems most likely that the largely equivalent performance for the RE and LE and the right and left visual fields is accomplished by almost equally proficient, though different, processing mechanisms. Thus, it appears more probable that the mechanism by which processing is accomplished, rather than the gross accuracy of the process itself, distinguishes the performance of the right and left hemispheres on tests of perceptual asymmetry. A delineation of these processes may prove a more productive avenue of exploration for those interested in defining the underlying determinants of perceptual asymmetries. Such investigations may, in addition, lead to a method that can identify the localization of functions within the brain more reliably. However, until such a method is elaborated, it would seem most appropriate to conclude that the perceptual advantages ob- tained with typical divided or focused attention procedures provide little direct information about functional hemispheric asymmetries.

\section{REFERENCES}

Bloch, M., Hellige, J. (1989). Stimulus intensity, attentional instructions, and the ear advantage during dichotic listening. Brain \& Cognition, 9, 136-148.

BRYDEN, M. P. (1978). Strategy effects in the assessment of hemispheric asymmetry. In G. Underwood (Ed.), Strategies of infomation processing (pp. 117-149). London: Academic Press.

BRYDEN, M. P. (1982). Laterality: Functional asymmetry in the intact brain. London: Academic Press.

BRYDEN, M. P. (1988). An overview of the dichotic listening procedure and its relation to cerebral organization. In K. Hugdahl (Ed.), Handbook of dichotic listening (pp. 1-45). Toronto: Wiley.

Bryden, M. P., Free, T., Gagne, S., aroff, P. (1991). Handedness effects in the detection of dichotically-presented words and emotions. Cortex, 27, 229-235.

Bryden, M. P., Hécaen, H., DeAgostini, M. (1983). Patterns of cerebral organization. Brain and Language, 20, 249-262.

Bryden, M. P., MACRAE, L. (1988). Dichotic laterality effects obtained with emotional words. Neuropsychiatry, Neuropsychology, and Behavioural Neurology, 1, 171-176.

Bryden, M. P., Munhall, K., \& Allard, F. (1983). Attentional biases and the right-ear effect in dichotic listening. Brain \& Language, 18, 236-248

Bryden, M. P., Murray, J. (1985). Toward a model of dichotic listening performance. Brain \& Cognition, 4, 241-257.

Bryden, M. P., Sprott, D. A. (1981). Statistical determination of degree of laterality. Neuropsychologia, 19, 571-581.

DeAN, R., Hu^, M. (1982). Laterality effects in cued auditory asymmetries. Neuropsychologia, 20, 685-690.

GoODGlASs, H. (1967). Binaural digit presentation and early lateralized brain damage. Cortex, 2, 295-306.

Hiscock, M., \&TEwarT, C. (1984). The effect of asymmetrically focused attention upon subsequent ear differences in dichotic listening. Neuropsychologia, 22, 337-351.

Hugdahl, K. , ANDersson, L. (1986). The "forced-attention paradigm" in dichotic listening to CV syllables: A comparison between adults and children. Cortex, 22, 417-432.

KallmaN, H. J. (1978). Can expectancy explain reaction time ear asymmetries. Neuropsychologia, 19, 571-581.

KIMURA, D. (1961a). Cerebral dominance and the perception of verbal stimuli. Canadian Journal of Psychology, 15, 166-171

KimURA, D. (1961b). Some effects of temporal lobe damage on auditory perception. Canadian Journal of Psychology, 15, 156-165.

KinsBourne, M. (1975). The mechanism of hemispheric control over the lateral gradient of attention. In P. M. A. Rabbitt \& S. Dornic (Eds.), Anention and Pefformance V (pp. 81-97). London: Academic Press.

Ley, R. G., \& BRYden, M. P. (1982). A dissociation of right and left hemispheric effects for recognizing emotional tone and verbal content. Brain \& Cognition, 1, 3-9.

Mondor, T. A., BRYden, M. P. (1991). The influence of attention on the dichotic REA. Neuropsychologia, 29, 1179-1190.

Mondor, T. A., \& Bryden, M. P. (1992a). On the relation between visual spatial attention and visual field asymmetries. Qwarterly Journal of Experimental Psychology: Human Perception \& Performance, 4AA, 529-555.

Mondor, T. A., \& BRyden, M. P. (1992b). Orienting of auditory spatial attention: Effects of a lateralized tone cue. Neuropsychologia, 29, 1179-1190.

Müller, H. J., \& RABbitT, P. M. (1989). Reflexive and voluntary orienting of visual attention: Time course of activation and resistance to interruption. Journal of Experimental Psychology: Human Perception \& Performance, 15, 315-330. 
Obrzut, J., Conrad, P., Bryden, M. P., Bollek, C. (1988). Cued dichotic listening with right-handed, left-handed, bilingual and learningdisabled children. Neuropsychologia, 26, 119-131.

REPP, B. (1976). Identification of dichotic fusions. Journal of the Acoustical Society of America, 60, 456-469.

SidTIS, J. J. (1982). Predicting brain organization from dichotic listening performance: Cortical and sub-cortical functional asymmetries contribute to perceptual asymmetries. Brain \& Language, 17, 287-300.

Sidtis, J. J. (1984). Brain asymmetries large and small. Brain \& Language, 21, 354-357.

SIDTIS, J. J. (1988). Dichotic listening after commissurotomy. In K. Hugdahl (Ed.), Handbook of dichotic listening (pp. 161-184). Toronto: Wiley.

SPRINGer, S. P., Deutsch, G. (1989). Left brain, right brain. New York: W. H. Freeman.

WEXLER, B., \& HalWEs, T. (1981). Right eat bias in the perception of loudness of pure tones. Neuropsychologia, 19, 147-150.

WEXLER, B., HALWES, T. (1983). Increasing the power of dichotic methods: The fused thymed words tests. Neuropsychologia, 21, 59-66.

YANTIS, S., \& JoNIDES, J. (1990). Abrupt visual onsets and selective attention: Voluntary versus automatic allocation. Journal of Experimental Psychology: Human Perception \& Performance, 16, 121-134.

ZatorRe, R. (1989). Perceptual asymmetry on the dichotic fused words test and cerebral speech lateralization determined by the carotid amytal test. Neuropsychologia, 27, 1207-1219.

\section{NOTES}

1. We use the term bias to indicate a skewing of the distribution of attention. For example, an attentional bias toward the RE is meant to indicate that the distribution of attentional resources is skewed toward the RE.

2. It is true that in the typical dichotic listening study, subjects are asked to attend to one ear for a block of several trials. Clearly, such instructions rely heavily on the subjects' cooperation. In addition, however, it may be that subjects find it more difficult to pay attention to one ear for many trials in succession and that this effect also contributes to any obtained ear advantages. The precuing technique clearly attenuates the impact of this possible effect, since subjects do not have to maintain their attention on one ear for a long period of time.

3. These stimuli were the same as those used by Bryden and MacRae (1988) and by Bryden et al. (1991).

(Manuscript received November 7, 1991; revision accepted for publication March 20, 1992.) 